\title{
Landau damping via the harmonic sextupole
}

\author{
Lidia Tosi, Victor Smaluk, and Emanuel Karantzoulis \\ Sincrotrone Trieste, Trieste, Italy \\ (Received 25 November 2002; published 14 May 2003)
}

\begin{abstract}
Multibunch instabilities of a storage ring electron beam occur due to coherent particle oscillations generated through a bunch to bunch coupling via the impedances, deteriorating the beam quality. One cure for multibunch instabilities is Landau damping, i.e., introducing a spread in the oscillation frequencies among the particles of the individual bunches in order to destroy the coherence of the coupled multibunch oscillation. Measurements at ELETTRA have shown that the harmonic sextupole provides Landau damping capable of suppressing transverse multibunch instabilities. The damping is induced by the nonlinear tune spread with amplitude among the electrons within the individual bunches.
\end{abstract}

DOI: 10.1103/PhysRevSTAB.6.054401

PACS numbers: 29.20.-c, 29.27.Bd

\section{INTRODUCTION}

The presence of multibunch instabilities is one of the most common reasons for beam quality deterioration and current limitation in storage rings. The instability occurs due to coherent oscillations generated through a bunch to bunch coupling via a long memory structure in the storage ring (e.g., via an rf cavity). Every bunch may leave, while it passes through the structure, an electromagnetic field which perturbs the motion of the following bunches. When the system gets this loop closed (generally after some revolutions), all bunches execute a coupled multibunch oscillation coherently with a certain phase difference between them. One cure for multibunch instabilities is to introduce a spread in the oscillation frequencies among the particles of the individual bunches in order to destroy the coherence of the coupled multibunch oscillation. This mechanism is well known in many branches of physics as Landau damping, whereby a collective motion performed by a certain number of particles is damped by an increasing spread in the oscillation frequencies of the individual particles. As the coherent oscillations build up a center of mass motion, the latter starts decreasing as particles go out of phase with respect to each other and decohere. Individual particles may be still oscillating but the center of mass motion is damped. If the driving force depends on the center of mass, then it too will disappear and in synchrotron storage rings the amplitudes of the individual particles of the bunches will decrease with the betatron damping rate.

The necessary spread in the transverse plane may be provided by introducing octupole magnets in the lattice (which are not present in ELETTRA [1]) or by setting nonzero chromaticities via sextupoles in the dispersive arcs. While the sextupoles give rise to tune spreads with momenta, the octupoles generate tune shifts with amplitude as a first-order effect. However sextupoles induce also tune shifts with amplitude as a higher order effect that may nevertheless induce an efficient spread.
This paper is about to examine and to present measurements based on Landau damping induced by the nonlinear behavior of the sextupole magnets. Theoretically the tune shift with amplitude can be obtained by applying perturbation theory to the Hamiltonian describing the dynamics in the action and angle variables [2]. Only the tune shifts with amplitude due to second order effects of the sextupoles will be considered here. These may be expressed according to

$$
\begin{aligned}
& \Delta \nu_{x}=C_{11} \cdot 2 J_{x}+C_{12} \cdot 2 J_{y}, \\
& \Delta \nu_{y}=C_{12} \cdot 2 J_{x}+C_{22} \cdot 2 J_{y},
\end{aligned}
$$

where the subscripts $x$ and $y$ denote the horizontal and vertical planes, respectively, $\Delta \nu(u=x, y)$ are the nonlinear tune shifts, $J_{u}$ the perturbed action variables, and $C_{11}, C_{12}, C_{22}$ are coefficients which depend on the sextupole strengths, on the beta functions at their locations, and on the relative phase advances between them [2,3]. As the units of $J_{u}$ are meters, those of the coefficients are meters $^{-1}$. The lattice of the Italian third generation light source ELETTRA has been provided with three sextupole families. While two of these families are placed in the dispersive arcs to compensate for the fairly large natural chromaticities (defined as $\xi=[(d \nu) /(d p / p)]$ ), the third family $S 1$ is a harmonic sextupole located in the dispersive free regions. It has been introduced to enlarge the dynamic aperture by compensation of the geometric aberrations generated by the chromatic sextupoles. Thus, it is possible to change the nonlinear configuration of the machine (hence the tune shifts with amplitude) by acting on its strength, maintaining at the same time constant values for the chromaticities. Figure 1 shows the theoretical behavior of the three coefficients as functions of the harmonic sextupole normalized strength $K_{2} L$ at $2.0 \mathrm{GeV}$ (nominal value is $2.11 \mathrm{~m}^{-2}$ ). The sextupole normalized strength is defined as $K_{2} L=$ $(L / B R)\left(\partial^{2} B_{y} / \partial x^{2}\right)$. From the figure, one immediately 


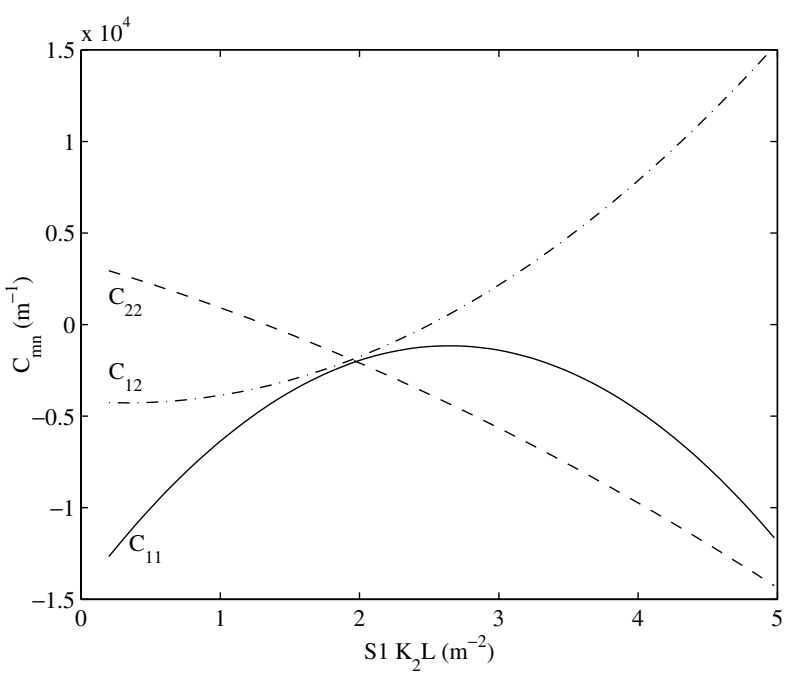

FIG. 1. $C_{11}, C_{12}$, and $C_{22}$ vs the harmonic sextupole normalized strength at $2.0 \mathrm{GeV}$.

sees that the effect of the harmonic sextupole on the tunes may be rather strong. One may expect an enhancement of horizontal instabilities at the strength corresponding to the minimum of the absolute value of $C_{11}$ and of the vertical ones at the zero crossing of $C_{22}$. Observations and measurements confirm that in ELETTRA the harmonic sextupole $S 1$ plays an essential role in damping coherent transverse multibunch instabilities, to the extent that they can even be totally suppressed when the sextupole is appropriately set [4]. Many measurements have been performed and they all indicate a strong correlation with the behavior of the coefficients $C_{11}$ and $C_{22}$ [5-7].

As a basic tool for the measurements, the digital transverse multibunch feedback (TMBF) [8] of ELETTRA has been used. It consists of a wide-band bunch-by-bunch system where the positions of each of the 432 bunches can be acquired. The wide-band signals of the bunches, separated by $2 \mathrm{~ns}$, are demodulated into a base-band signal between 0 and $250 \mathrm{MHz}$. The memory of the system is such that it allows the acquisition of the bunch positions in real time over a time span of $192 \mathrm{~ms}$ and thus over 200000 turns, yielding a precious tool for the analysis of the details of the behavior of the single bunches. It has to be however underlined that at the moment the acquired signals cannot be converted to the actual beam position because the system is still not calibrated.

This paper presents the main results of the observations, of the measurements performed, and of computer simulations of the different Landau damping rates induced by the variations of the strength of the harmonic sextupole. Although similar observations have been made for the vertical plane, due to the relatively simpler mechanism, both measurements and simulations presented are related to the horizontal plane. In this case, the coupling among the two planes due to the sextupoles is eliminated and the tune shift with amplitude may be reduced to
$\Delta \nu_{x}=C_{11} \cdot 2 J_{x}$ provided that the collective motion is principally in the horizontal plane. Section II discusses the fast coherent damping observation when kicking a single bunch at the injection energy of $0.9 \mathrm{GeV}$. Some results regarding the vertical plane done at a macroscopic level, namely, not using the TMBF, are presented in Sec. III, where the effects of the strength of $S 1$ on general machine parameters are presented. Section IV instead presents the results of the damping effects measured with the TMBF compared to simulations when coupled horizontal multibunch instabilities are present.

\section{LANDAU DAMPING OBSERVATION}

Effects of nonlinearities induced by the harmonic sextupole on horizontal betatron motion have been measured in a single bunch and compared with computer simulation. Coherent betatron motion was excited using the injection kickers and turn-by-turn data were taken using the TMBF system. There is a theoretical analysis [9] of nonlinear beam dynamics in the case of free betatron oscillation excited by a short kick and observed using a photomultiplier tube with a blind placed in the image plane. With some modifications this analysis can be also applied to a beam position monitor (BPM) which is the pickup of the TMBF.

A single bunch in the absence of coherent betatron and synchrotron oscillations has a particle distribution function in betatron phase space represented in the actionphase variables as

$$
f(J, \varphi, t)=\sum_{n=-\infty}^{\infty} f_{n}(J, t) e^{-i n(\omega t-\varphi)}
$$

where $J=\left(a^{2} / 2 \beta\right)$ is the perturbed action, $a$ is the oscillation amplitude, $\varphi$ is the phase, $\omega=2 \pi \nu f_{\text {rev }}$ is the betatron frequency, and $\beta$ is the beta function. If $G(x, \omega)$ is the transfer function of a diagnostic device, the output signal is proportional to

$$
X=\sum_{k=-\infty}^{\infty} \sum_{n=-\infty}^{\infty} e^{-i\left(k \omega_{0}-n \omega\right) t} \int_{0}^{\infty} f_{n}(J, t) K_{k n} d J
$$

where $\omega_{0}=2 \pi f_{\text {rev }}$ and $K_{k n}=\int_{-\pi}^{\pi} G\left(a \cos \varphi, k \omega_{0}\right) e^{i n \varphi} d \varphi$. Note that $f_{n}(J, t)$ is a slow function of time. Supposing that the transfer function of the BPM electronics is linear and the amplitude-phase characteristics are constant in the frequency band where the betatron harmonics have considerable amplitudes (usually $n<5$ to 10 ), then $K_{k n}=$ $K=$ const, and the signal of the $n$th betatron harmonic is proportional to

$$
X_{n}=K e^{-i\left(\omega_{0}-n \omega\right) t} \int_{0}^{\infty} f_{n}(J, t) d J+\text { c.c. },
$$

with the envelope as 


$$
A_{n}=K\left|\int_{0}^{\infty} f_{n}(J, t) d J\right|
$$

If the beam current is small and collective effects are negligible, as in the case of zero chromaticity, a first-order approximation gives

$$
f_{n}(J, t)=f_{n}(J, 0) e^{-i n(\partial \omega / \partial J) J t} .
$$

Here $(\partial \omega / \partial J)=2 \omega_{0} C$, where $C$ is the nonlinear coefficient $C_{11}$ (1) for the horizontal plane or $C_{22}$ for the vertical one. Placing (6) into (5) gives

$$
A_{n}=K\left|\int_{0}^{\infty} f_{n}(J, 0) e^{-i n(\partial \omega / \partial J) J t} d J\right| .
$$

In the case of nonzero chromaticity, instead of (6) the distribution function is

$$
\begin{aligned}
f_{n}(J, t) & =e^{-i n(\partial \omega / \partial J) J t} \int_{0}^{\infty} \int_{0}^{2 \pi} f_{n}(J, \varepsilon) e^{-i n(\partial \omega / \partial E)(\varepsilon / \Omega)[\sin (\psi-\Omega t)-\sin \psi]} \varepsilon d \varepsilon d \psi \\
& =e^{-i n(\partial \omega / \partial J) J t} \cdot 2 \pi \int_{0}^{\infty} f_{n}(J, \varepsilon) J_{0}\left(2 n \frac{\partial \omega}{\partial E} \frac{\varepsilon}{\Omega} \sin \frac{\Omega t}{2}\right) \varepsilon d \varepsilon
\end{aligned}
$$

where $(\partial \omega / \partial E)=\left(\omega_{0} / E\right) \xi, J_{0}$ is the Bessel function, and $\Omega, \varepsilon, \psi$ are the frequency, amplitude, and phase of the synchrotron oscillation. If $f_{n}(J, \varepsilon)$ can be represented as a product $f_{n}(J, \varepsilon)=F_{n}(J) \phi(\varepsilon)$, then, to take the chromaticity into account, $f_{n}(J, t)$ in (6) should be multiplied by

$$
M_{n}=2 \pi \int_{0}^{\infty} \phi(\varepsilon) J_{0}\left(2 n \frac{\partial \omega}{\partial E} \frac{\varepsilon}{\Omega} \sin \frac{\Omega t}{2}\right) \varepsilon d \varepsilon .
$$

In this case $A_{n}(t)$ is modulated with the modulation parameter $M_{n}(t)$.

In our experiments a coherent betatron oscillation was excited by a short kick. If $F(J)$ is the distribution function of oscillation amplitudes before the kick, then after the kick the distribution function becomes

$$
f(J, \varphi, \varepsilon)=F(J-2 \sqrt{J \delta J} \sin \varphi+2 \delta J) \phi(\varepsilon),
$$

where $\delta J=\left(\delta a^{2} / 2 \beta\right)$ is the action perturbation due to the kick with $\delta a$ amplitude. Calculating the first betatron harmonic $A_{1}(t)$ with the conditions $\delta a \gg \sigma_{\perp}$ and $t \ll$ $\left(\omega_{0} C \sigma_{\perp}^{2} / \beta\right)^{-1}$ where $\sigma_{\perp}$ is the transverse beam size gives

$$
A_{1}(t)=M_{1}(t)\left|\int_{0}^{\infty} F(J) J_{0}\left(2 \omega_{0} C \sqrt{J \delta J} t\right) d J\right| .
$$

For a stable bunch, the distribution functions $F(J)$ and $\phi(\varepsilon)$ are Gaussian and, according to (11), the envelope is

$$
A_{1}(t) \propto e^{-\left(t^{2} / 2 \tau^{2}\right)} e^{-\left[(\partial \omega / \partial E)\left(\sigma_{E} / \Omega\right)\right]^{2}(1-\cos \Omega t)},
$$

where $\tau=\left(2 \omega_{0} C \sigma_{\perp} \delta a / \beta\right)^{-1}$ and $\sigma_{E}$ is the rms energy spread. Thus, if the chromaticity is zero, the envelope of betatron oscillation is Gaussian.

Figure 2 shows an example of measured data in comparison with simulation. The measurement was performed on a single bunch at $0.9 \mathrm{GeV}$ energy with a beam current of $1 \mathrm{~mA}$ and a chromaticity of 0.1 . Since the emittance at $0.9 \mathrm{GeV}$ is $1.415 \mathrm{~nm}$ rad and in the simulation $C_{11}$ is $-2835 \mathrm{~m}^{-1}$, the tune spread of the initial beam is $8 \times 10^{-6}$. The simulation was carried out for 1000 particles and the kick amplitude of almost $6 \mathrm{~mm}$ was fit to get the damping time close to the measured one because the measurement system is not calibrated. In the simulation plot one can see that the coherent oscillation damps and the incoherent one (i.e., beam size) grows. This effect is due to the mismatching of the particles' phases caused by the nonlinear tune spread. The initial beam size due to the decoherence grows up to approximately 0.7 of the initial oscillations when the motion becomes completely incoherent. At a later stage, the size will slowly decrease due to the natural damping. This is not shown in the figure since the damping time is $112 \mathrm{~ms}$.

The damping time was measured with the various harmonic sextupole strength in the $1.4-3.6 \mathrm{~m}^{-2}$ range. Figure 3 shows the measured damping rate versus the harmonic sextupole strength in comparison with the simulation data and with the theoretical curve calculated using the $\tau=\left(2 \omega_{0} C_{11} \sigma_{\perp} \delta a / \beta\right)^{-1}$ formula. One can see that the damping rates obtained by the measurements, the simulations, and the theory all agree very well. The parabolic shape of the damping rate graph repeats the behavior of a cubic nonlinearity parameter and, namely, the harmonic sextupole acts as an octupole. The Landau damping of coherent betatron oscillation is rather strong,
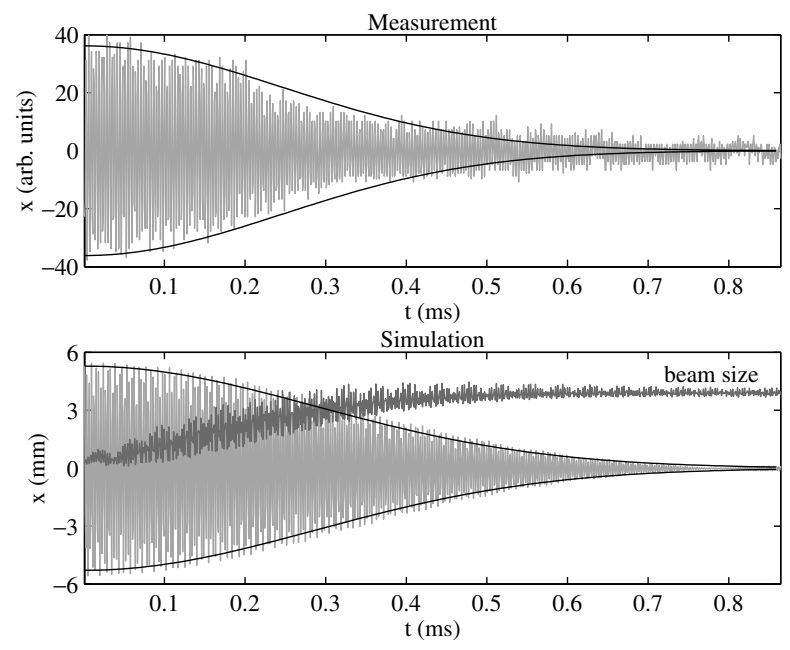

FIG. 2. Gaussian damping of the coherent oscillation. 


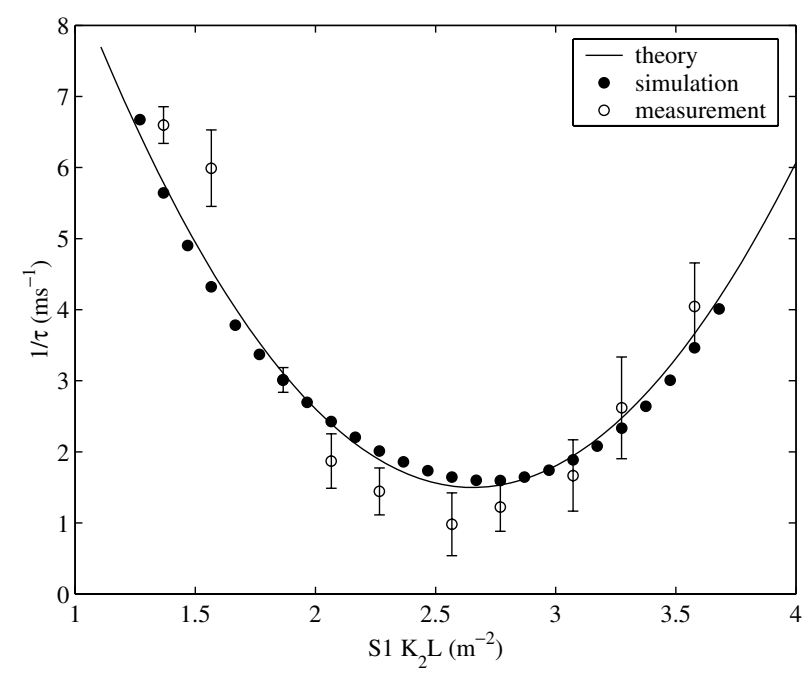

FIG. 3. Damping rate versus harmonic sextupole strength.

with $\tau=0.2$ to $1 \mathrm{~ms}$ with the nominal small positive chromaticity $(\sim 0.1)$.

\section{MACROSCOPIC INSTABILITY MEASUREMENTS}

The energy loss per turn of the electrons is compensated in ELETTRA by four rf cavities located in the dispersive arcs. The higher order modes (HOM) of the cavities are controlled by setting the temperatures of the cavities [10]. In particular, the behavior of the horizontal coupled bunch (dipole) mode HCBM 318, driven by the cavity HOM T3 [11], versus the harmonic sextupole strength was investigated, as well as that of the HCBMs 414-417 whose origin is still a matter of investigation. Figure 4 compares the theoretical values of the coefficient $C_{11}$ with the amplitude of HCBM 318 measured with a spectrum analyzer as a function of the harmonic sextupole strength. The mode could be totally suppressed at $2.0 \mathrm{GeV}$ by setting the sextupole below $1.3 \mathrm{~m}^{-2}$ or above

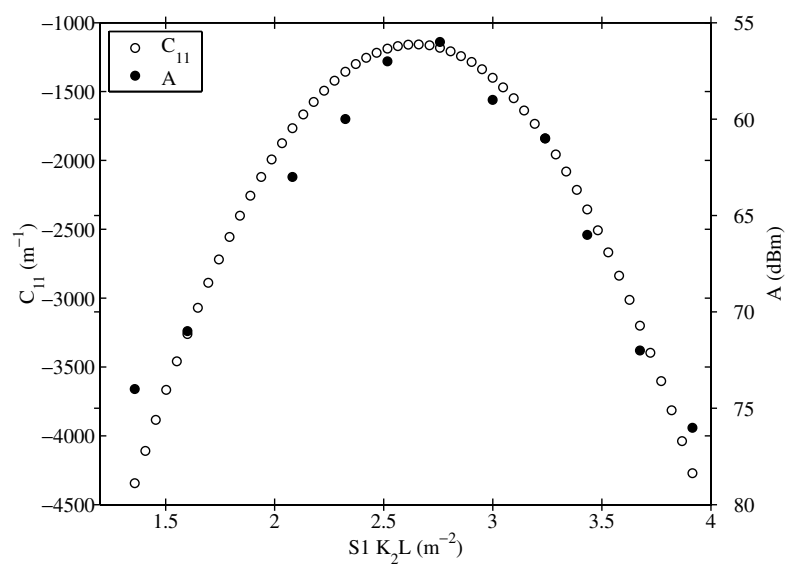

FIG. 4. Comparison of the amplitude of HCBM 318 with $C_{11}$ as functions of the harmonic sextupole strength.
$4.1 \mathrm{~m}^{-2}$. The same parabolic behavior as $C_{11}$ was found for the excitation levels of HCBM 414, although in this case the two values of the sextupole strength for which the mode was totally suppressed were found to be different. While the peak of the excitation level of the modes was always found to be around the sextupole strength of $2.75 \mathrm{~m}^{-2}$ (depending on the optics), deeper investigations revealed that the two boundary values of the sextupole at which the modes can be suppressed depend on the beam current, on the modes excited, on the filling pattern, and on the presence or less of longitudinal instabilities. It was noticed that the stronger the mode, the higher the beam current filling percentage and the lower the longitudinal excitations were, the wider was the range of the sextupole strength for which the modes were excited. This, namely, translates into requiring higher absolute values for $C_{11}$ in order to achieve the suppression of the horizontal modes. While the dependence on the beam current and on the particular mode excited reflects the "strength" of the driving force, the one on the filling pattern and on the presence or less of longitudinal modes is due to how well the driving force manages to couple to the beam's modes. In fact the coupling efficiency of a transverse driving force may be diminished by a gap within the bunch train, by the different arrival times of the bunches, and possibly also on the longitudinal density of the bunches.

It has to be strongly underlined that even in the total absence of longitudinal modes, confirmed by measurements of the full longitudinal spectrum, it was found that the amplitudes of the excited transverse modes follow the same trend of $C_{11}$ with the harmonic sextupole strength, excluding thus any interference of longitudinal modes in the mechanism by which the sextupole may be influencing the dynamics. This was a particularly important point to confirm, because the cavities are located in the dispersive arc. Furthermore, it was found, by measuring full longitudinal spectra as a function of the harmonic sextupole strength, that the excitation level of any longitudinal instability did not depend on the sextupole.

The major beam parameters, such as tunes, closed orbit, dispersion, and chromaticities, were measured as a function of the harmonic sextupole strength. No significant changes were noticed in these quantities, with the exception of the horizontal chromaticity, which for the measurement of Fig. 4 was found to gradually increase from 0.1 for the sextupole strength of $1.3 \mathrm{~m}^{-2}$ to 1.6 for $4.1 \mathrm{~m}^{-2}$. Both settings are values for which the mode was suppressed. This variation of the chromaticity is not expected to be the major cause for the suppression of the mode. In fact, no change in the excitation level of the mode was noticed when the horizontal chromaticity was set to 1.6 , by using the chromaticity correcting sextupoles. Since however the value of $C_{11}$ also depends on the strengths of the chromatic sextupoles, investigations were carried out in order to confirm the leading role of $C_{11}$ in the phenomenon against that of the horizontal 
chromaticity. The results revealed that the suppression of the excited modes using the chromaticity correcting sextupoles required a horizontal chromaticity greater than 7 . The values instead of $C_{11}$ found for these settings of the chromatic sextupoles were computed to be identical to those at which $S 1$ suppresses the mode.

Associated with the presence of the transverse modes, low frequencies in the spectrum (typically below $100 \mathrm{~Hz}$ ) have also been observed. Whereas in some situations there appears a well-defined peak at low frequency, in others the whole background noise level increases by $1-2$ orders of magnitude, according to whether only one or more modes were present, respectively. Whenever well-defined low frequencies were noticed, measurements on the spectrum analyzer in zero span mode of the unstable sideband showed periodicities corresponding to the peaks measured in the low frequency spectrum range. Detailed investigations in a narrow frequency range around the unstable sideband with an appropriate sweeping time multiple of the associated low frequency showed a fine structure indicating that the sideband was moving with the measured low frequency. As it will be shown in the following section, the apparent movement of the unstable sideband is related to an increasing spread in the frequencies of the particles within the single bunches. The strength of the harmonic sextupole also influences the values of the low frequencies. Figure 5 shows the dependence of the low frequencies both on the harmonic sextupole strength and on the beam current for a fixed setting of the sextupole at $2.75 \mathrm{~m}^{-2}$. The low frequencies fit extremely well to a second order polynomial as a function of the sextupole, presenting a maximum where the absolute value of $C_{11}$ is minimum.

Another measurement that was considered necessary was to confirm that effectively the horizontal tune shift with amplitude $\Delta \nu_{x}$ follows the same behavior as $C_{11}$. For this purpose, the horizontal tune spread of a $1 \mathrm{~mA}$ beam in single bunch mode was measured as a function of the sextupole strength, by exciting the beam with a constant amplitude using the spectrum analyzer of the tune measurement system. A good parabolic agreement was found with the minimum at $K_{2} L=2.75 \mathrm{~m}^{-2}$.
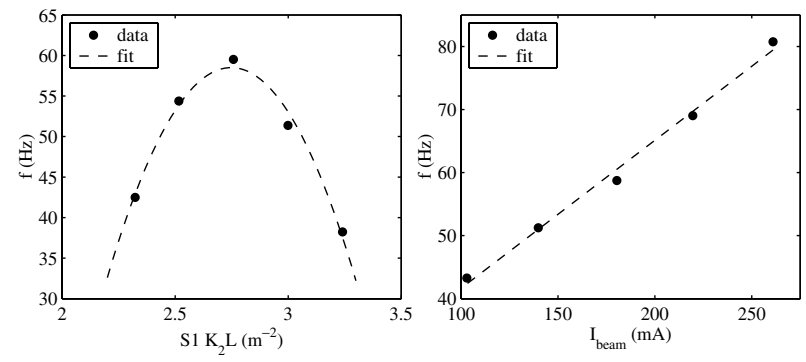

FIG. 5. Low frequencies associated with transverse instabilities as functions of the harmonic sextupole strength (left panel) and of beam current (right panel) for a fixed strength of the sextupole $\left(K_{2} L=2.75 \mathrm{~m}^{-2}\right)$.
TABLE I. Amplitudes of vertical modes versus harmonic sextupole strength.

\begin{tabular}{ccccc}
\hline \hline $\begin{array}{c}K_{2} L \\
\left(\mathrm{~m}^{-2}\right)\end{array}$ & $\begin{array}{c}\text { VCBM 167 } \\
(\mathrm{dB})\end{array}$ & $\begin{array}{c}\text { VCBM 246 } \\
(\mathrm{dB})\end{array}$ & $\begin{array}{c}\text { VCBM 344 } \\
(\mathrm{dB})\end{array}$ & $\begin{array}{c}\text { VCBM 422 } \\
(\mathrm{dB})\end{array}$ \\
\hline 1.38 & 16.7 & 16.3 & 17.0 & 37.3 \\
1.66 & 18.6 & 10.8 & 11.0 & 38.7 \\
4.17 & 6.1 & $\cdots$ & $\cdots$ & 25.4 \\
\hline \hline
\end{tabular}

Measurements at a macroscopic level were carried out also for the vertical plane. Assuming the same hypothesis as for the horizontal plane, some preliminary studies have been made on the dependence of vertically excited modes on $C_{22}$. As can be seen in Fig. $1, C_{22}$ presents very low values in the range of strength for the harmonic sextupole between $0.9 \mathrm{~m}^{-2}$ and $1.8 \mathrm{~m}^{-2}$, becoming fairly large for strength above $2.75 \mathrm{~m}^{-2}$. Thus, if the hypothesis that the coefficients in the tune shift with amplitude is the leading mechanism by which excitation levels of the instabilities depend on the harmonic sextupole, one would expect an enhancement of the vertical modes for lower strength of the sextupole and suppression for higher ones. Table I illustrates the amplitudes of the modes as a function of the strength. In particular, during the measurement, it was noticed that trying to set the harmonic sextupole to $1.25 \mathrm{~m}^{-2}\left(C_{22}=0\right.$ for $\left.1.20 \mathrm{~m}^{-2}\right)$, the vertical instability grew extremely strong with an evident vertical beam size blowup on the synchrotron light beam profile monitor and subsequent loss of $100 \mathrm{~mA}$ of beam current. Missing data for the vertical amplitudes are due to the coexistence in this range for the sextupole strengths of horizontal modes which were suppressing the vertical ones. The suppression of the vertical modes during the existence of the horizontal ones is in agreement with an influence of the coupling term $C_{12}$.

\section{INSTABILITY MEASUREMENTS WITH THE TMBF}

Coupled multibunch instabilities can be viewed as consisting of two collective motions: a macroscopic one among the centroids of the individual bunches and a microscopic one among the single particles within the individual bunches. Thus, in the past, based on the results of the previous section when observations were not facilitated by the TMBF, an important question arose: whether the tune spread induced by the harmonic sextupole acted on different bunches or on the particles within the individual bunches. Simulations done in 1999 using a macroscopic model of the bunches could reproduce only qualitatively the main features of the phenomena, but not quantitatively. When the TMBF became operational, allowing the visualization of the center of mass motion of individual bunches, further exhaustive measurements were performed for the understanding of the mechanism involved. 

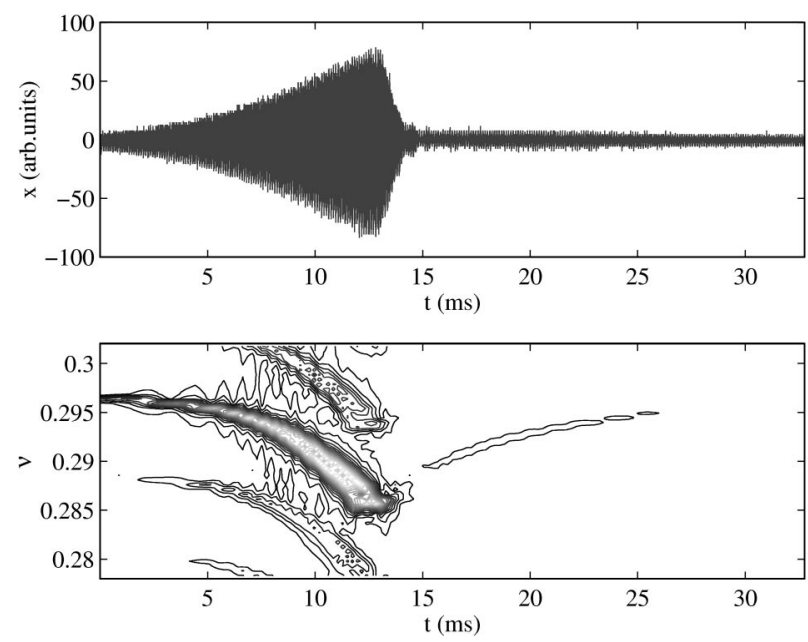

FIG. 6. Horizontal instability for $K_{2} L=2.3 \mathrm{~m}^{-2}$.

Figure 6 shows the measured center of mass motion, together with its spectrum (tune resolution is $5 \times 10^{-4}$ ), of one of the bunches undergoing the horizontal coupled multibunch instability HCBM 318 with $S 1$ set not far from the minimum value for the tune shift with amplitude. Setting $S 1$ to $2.75 \mathrm{~m}^{-2}$ brought a significant beam loss.

The damping of the center of mass motion is not due to a simple detuning of the HOM. In fact, observing Fig. 7 where the same analysis is shown for the same bunch but with the sextupole set to a stronger tune shift with amplitude, one can note that the damping occurs at a much smaller tune shift of the center of mass. This occurs because of the higher damping efficiency due to the increase in the spectral density of the betatron tune distribution.

Although the two motions are qualitatively different they have one common feature: during the growth of the instability there is a broadening of the tune distribution
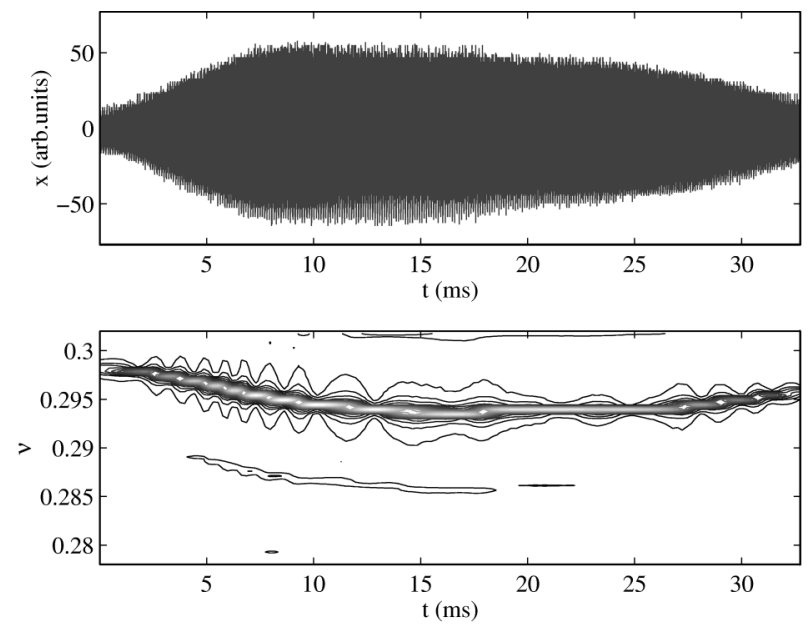

FIG. 7. Horizontal instability for $K_{2} L=1.8 \mathrm{~m}^{-2}$. width. This broadening cannot be due to the spread in amplitudes of the center of mass, because during the damping the spread in amplitudes is much larger, but the width is noticeably smaller. The only interpretation can be that there is an increasing tune spread within the coherent particles of the bunch, resulting as a tune shift of the center of mass together with a broadening. In fact, since amplitudes can be only positive, the tune shift with amplitude is unidirectional. Thus, if there is an increasing spread during the collective motion, then the tune of the center of mass will seemingly move at a rate that will generate a broadening in its spectrum. The same analysis has also been performed on all the other bunches for the same two sextupole strengths and they all simultaneously present similar features with identical tune shifts and broadenings. The low frequency oscillations mentioned in the previous section have been found to be related to the repetition of excitation and damping of the coherent motion. The two figures also show the presence of synchrotron sidebands which are due to a not completely longitudinal stable situation. These however do not grow in intensity as the betatron frequency does, but they remain constant during the motion. This is retained to be a further confirmation of the absence of longitudinal dynamics in the mechanism.

To check the above-mentioned conclusions, a simulation of the instability has also been done using multibunch multiparticle tracking with an interaction between the beam and the single rf cavity mode T3. The results for $K_{2} L=2.3 \mathrm{~m}^{-2}$ are shown in Fig. 8. Although the details of the center of mass motion may differ from reality, the tune behavior is similar to the one of the measured data shown in Fig. 6 with a broadening of the spectrum while the instability rises. The details of the center of mass motion in the simulations have been found to depend on the number of particles per bunch that were being simulated. The present is the result of 100 particles per bunch
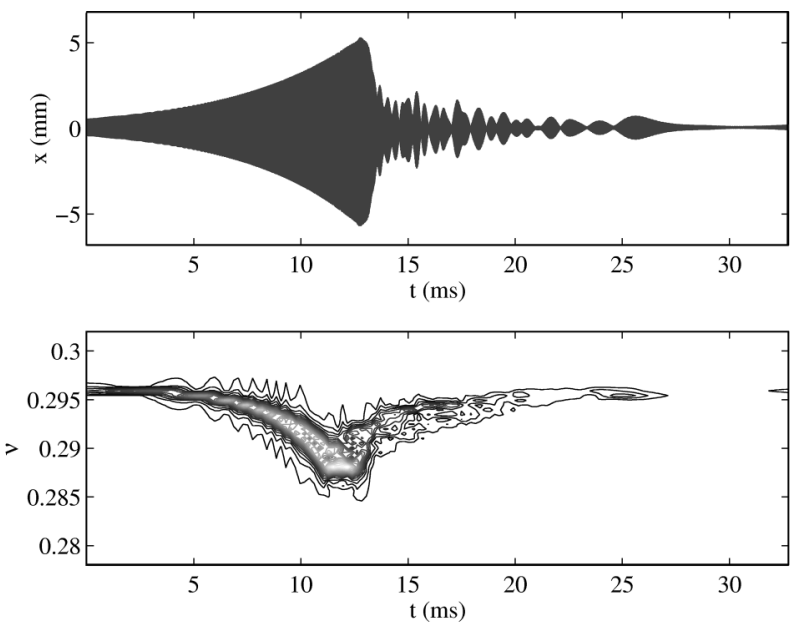

FIG. 8. Simulation of the horizontal instability for $K_{2} L=$ $2.3 \mathrm{~m}^{-2}$. 
and is surely still not sufficient to reproduce the reality faithfully, but the main essence of the phenomenon is there.

Knowing the measured rise time $(0.45 \mathrm{~ms})$ given by the HOM of the cavity, the betatron radiation damping time at $2.0 \mathrm{GeV}(10.21 \mathrm{~ms})$ and extracting the rise time of the bunch mode $(6 \mathrm{~ms})$ from Fig. 1, the Landau damping required to kill the instability is $\tau_{L}=0.512 \mathrm{~ms}$, provided by a tune spread of $\Delta \nu=\left(\pi f_{\text {rev }} \tau_{L}\right)^{-1}=5.37 \times 10^{-4}$. Assuming a constant chromatic tune spread, estimated for a stable beam and knowing the beam size at rest, the required amplitude for Landau damping results to be 1.8 times the beam size at rest, consistent with measurements from the synchrotron radiation beam profile monitor.

Multibunch analysis with the TMBF has been carried out also in the case in which several horizontal modes were present contemporaneously. Figure 9 shows a typical motion of an individual bunch in the presence of a horizontal multibunch instability with the spectrum shown in Fig. 10. The multibunch spectrum is obtained over 2000 turns in correspondence to the increase of the amplitude of the bunch oscillation. The main unstable modes are centered at 0.832 and $26.277 \mathrm{MHz}$ which correspond to the horizontal coupled mode numbers 417 and 394. Neither of the two modes correspond to known cavity higher order modes. Varying the harmonic sextupole changes the excitation level of the unstable modes with the maximum at $2.80 \mathrm{~m}^{-2}$ and a clearance of the modes below $1.65 \mathrm{~m}^{-2}$ and above $3.72 \mathrm{~m}^{-2}$.

The motion of individual bunches is very irregular and seemingly chaotic, due to the large number of modes involved, rendering its analysis as a function of the harmonic sextupole complicated. Thus the extraction of the rise times of an individual bunch motion is not sufficient to give an understanding of the mechanics involved, since these will depend heavily on the phases of the

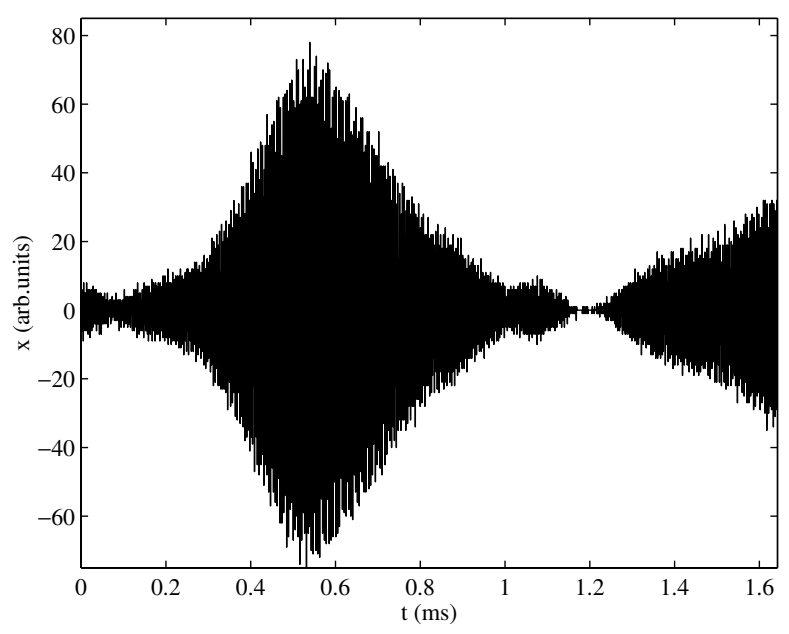

FIG. 9. Individual bunch motion.

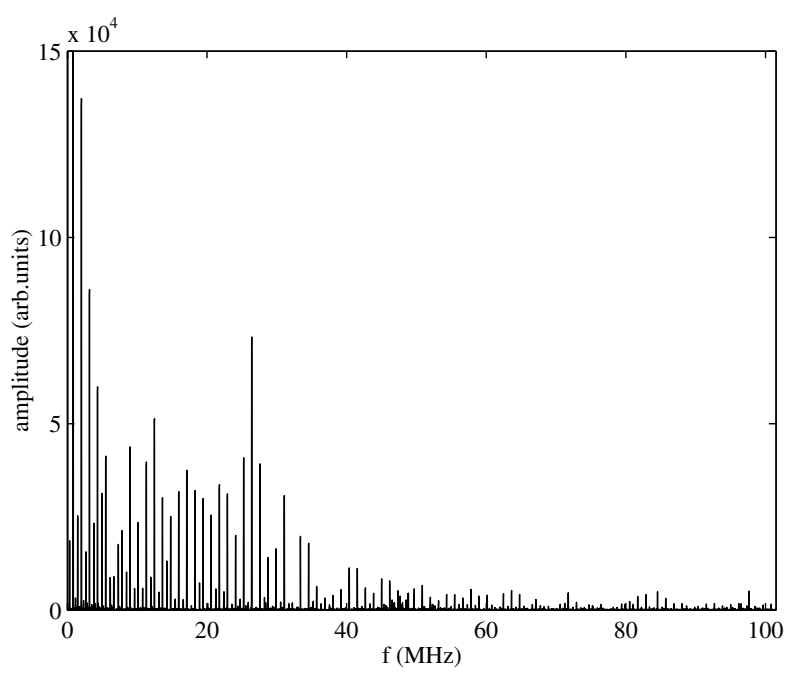

FIG. 10. Horizontal multibunch spectrum.

modes and on which one is most strongly influencing the motion at the moment. In this situation, an analysis of the evolution in time of the amplitudes of the unstable modes for various harmonic sextupole strength is more meaningful. It goes without saying that this was performed always during a time span in which horizontal beam motion was found to have increasing amplitudes. The rise and damping times of the unstable modes as a function of the harmonic sextupole strength are shown in Fig. 11. It can be observed that there is a concentration of unstable modes with positive rise times at those values for which the absolute value of $C_{11}$ is near its theoretical minimum. Furthermore, as the two sextupole strengths where the beam is stable are approached, a larger number of unstable modes decrease in amplitude and are damped.

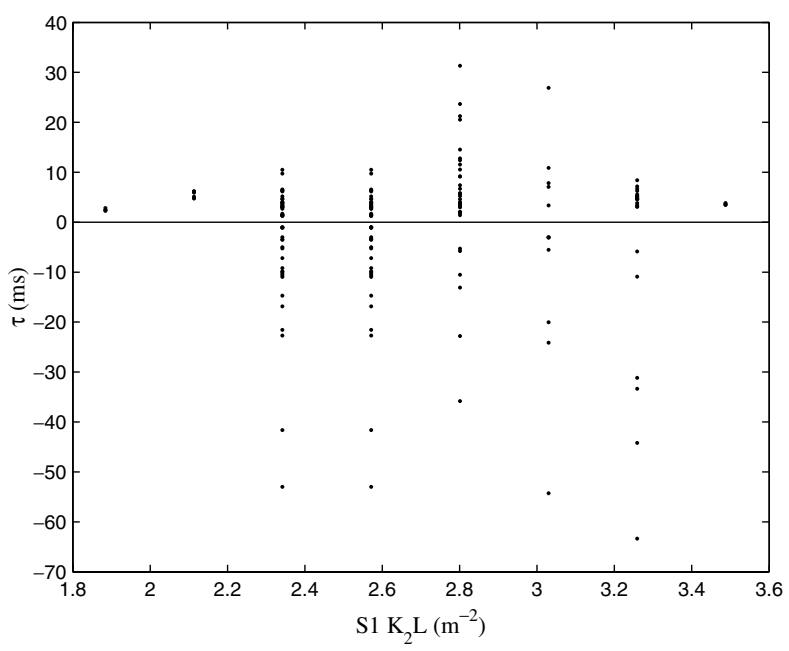

FIG. 11. Rise and damping times of the unstable modes as a function of the harmonic sextupole strength. 


\section{CONCLUSIONS}

At ELETTRA it has been found and shown that substantial damping against instabilities can be obtained by varying its harmonic sextupole. This by itself was not known. In fact, the reason of installing such a lens was to reduce the induced nonlinearities produced by the chromatic sextupoles and thus to enlarge the dynamic aperture. However it was not suspected that when varied, the change in the nonlinear tune shift with amplitude could be strong enough as to induce a sufficient damping to even suppress transverse coupled multibunch instabilities. Its damping efficiency is comparable to that of octupoles that generally are used for this purpose. Although settings of the harmonic sextupole which are different from its nominal one inevitably bring a reduction of the dynamic aperture of the machine and therefore its lifetime, the lifetime reduction is small compared to the benefits associated with the stabilization effects of the detuning.

Coming to the mechanism itself, it has been proven beyond any doubt that it is a pure Landau damping effect originating from the tune spread of the particles inside the individual bunches in the machine. The mechanism can therefore be effective both for multibunch and for single bunch operation. It is interesting to note that it has been observed as the typical Landau damping behavior. Namely, the damping efficiency increases with the increase of the frequency spectral density and the damping occurs when the center of mass frequency lies within the spread of the single particle frequencies.
At ELETTRA the harmonic sextupole is effectively used to damp efficiently horizontal coupled multibunch instabilities during operation for the users.

[1] ELETTRA Conceptual Design Report, Trieste, 1989.

[2] R. Nagaoka et al., Nucl. Instrum. Methods Phys. Res., Sect. A 302, 9-26 (1991).

[3] K. Y. Ng, Fermilab Report No. TM-1281, 1984.

[4] E. Karantzoulis, C. J. Bocchetta, A. Fabris, F. Iazzourene, M. Svandrlik, L. Tosi, and R. P. Walker, in Proceedings of the EPAC-98, Stockholm (IOP, Bristol, U.K., 1998).

[5] L. Tosi and E. Karantzoulis, in Proceedings of the PAC99, New York (IEEE, Piscataway, NJ, 1999).

[6] L. Tosi, V. Smaluk, D. Bulfone, E. Karantzoulis, and M. Lonza, in Proceedings of the PAC-2001, Chicago (IEEE, Piscataway, NJ, 2001).

[7] L. Tosi, V. Smaluk, and E. Karantzoulis, in Proceedings of the EPAC-2002, Paris (EPS-IGA/CERN, Geneva, 2002).

[8] D. Bulfone et al., in Proceedings of the DIPAC-2001, Grenoble (http://www.wsrf.fr/conferences/DIPAC/ DIPAC2001Proceedings.html).

[9] N. Vinokurov, V. Korchuganov, G. Kulipanov, and E. Perevedentsev, Chromaticity and Cubic NonLinearity Effects on a Dynamics Of Betatron Oscillation (Budker Institute of Nuclear Physics, Novosibirsk, 1976), pp. 76-87 (in Russian).

[10] M. Svandrlik et al., in Proceedings of the PAC-95, Dallas (IEEE, Piscataway, NJ, 1995).

[11] M. Svandrlik et al., in Proceedings of the EPAC-96, Sitges (IOP, Bristol, U.K., 1996). 